
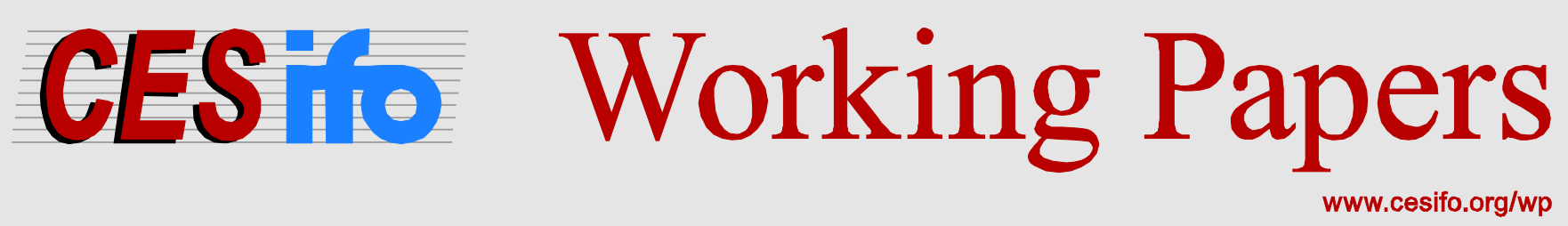

\title{
Robust Implementation of a Parsimonious Dynamic Factor Model to Nowcast GDP
}

\author{
Pablo Duarte \\ Bernd Süssmuth
}

\author{
CESIFO WORKING PAPER NO. 4574 \\ CATEGORY 12: EMPIRICAL AND THEORETICAL METHODS
}

JANUARY 2014

An electronic version of the paper may be downloaded

- from the SSRN website:

- from the RePEc website:

- from the CESifo website:

WwW.SSRN.com

Www.RePEc.org

www.CESifo-group.org/wp

\section{CESifo}




\title{
Robust Implementation of a Parsimonious Dynamic Factor Model to Nowcast GDP
}

\begin{abstract}
Quarterly GDP figures usually are published with a delay of some weeks. A common way to generate GDP series of higher frequency, i.e. to nowcast GDP, is to use available indicators to calculate a single index by means of a common factor derived from a dynamic factor model (DFM). This paper deals with the implementation stage of this practice. We propose a twotiered mechanism consisting in the identification of variables highly correlated with GDP as "core" indicators and a check of robustness of these variables in the sense of extreme bounds analysis. Accordingly selected indicators are used in an approximate DFM framework to exemplarily nowcast Spanish GDP growth. We show that our implementation produces more accurate nowcasts than both a benchmark stochastic process and the implementation based on the total set of core indicators.
\end{abstract}

JEL-Code: C380, C530.

Keywords: small-scale nowcasting models, Kalman Filter, extreme bounds analysis.

Primary Subjects [MSC]: 62M20, 62H25, 62M10.

\author{
Pablo Duarte \\ University of Leipzig \\ Grimmaische Str. 12 \\ Germany - 04109 Leipzig \\ duarte@wifa.uni-leipzig.de
}

\author{
Bernd Süssmuth \\ University of Leipzig \\ Grimmaische Str. 12 \\ Germany-04109 Leipzig \\ suessmuth@wifa.uni-leipzig.de
}

We thank participants of seminars at the University of Leipzig and of the IWH-CIREQ Macroeconometric Workshop on Forecasting and Big Data, especially Domenico Giannone, for comments and suggestions. 


\section{Introduction}

Monitoring economic activity on a monthly, weekly, or even higher frequency basis is at the heart of an early asessment of macroeconomic conditions and the crucial information base for decision making in the private and governmental sector. However, for industrialized economies in the contemporary world GDP data is published quarterly and information on GDP growth usually becomes available with a lag of six weeks seen from the end of a particular quarter through so-called flash or first releases 11 First releases are early announcements of second releases also referred to as final estimates (Camacho and Perez-Quiros, 2010; Camacho and Doménech, 2012). The latter are released about 14 weeks after the respective quarter has ended and are possibly subject to revisions and corrections. This circumstance has led several influential institutions such as the OECD to forecast growth rates of macroeconomic aggregates in real time and to make projections available on a more timely basis. Besides these "judgement based" real-time forecasts (Camacho and Perez-Quiros, 2010), monthly indicators are circulated that are drawn from official statistics or from surveys.

Relying on information from such data, which is available at the desired time and (higher) frequency and pre-selected to be closely related to the variable of interest, a dynamic factor model (DFM) can be used to deduce a common factor, that is, a single indicator at the higher frequency for the variable of interest. Recently, "approximate" DFMs have become a widely used tool to nowcast GDP growth at the national or supra-national level; see, for example, Aruoba, Diebold, and Scotti (2009) for the United States, Camacho and Doménech (2012) for Spain, Angelini et al. (2008a), Angelini et al. (2008b), Camacho and Perez-Quiros (2010), and Camacho and Garcia-Serrador (2013) for the Euro Area 22 They modify the "exact" DFM by Stock and Watson (1991) to account for problems of different frequency and asynchronous publication of series underlying the real-time forecast in applying a Kalman Filter strategy to fill up the series 3

A central critical aspect of this class of small-scale nowcasting models is the aforementioned pre-selection of indicators to be considered in the DFM routine. For example, Camacho and Perez-Quiros (2010, p. 672-674) suggest and apply the following selection procedure based on "successive enlargements." Having defined a set of core

\footnotetext{
${ }^{1}$ Note, the figure of six weeks represents just a rough average. As Castle, Fawcett, and Hendry (2009, p. 71) note preliminary estimates for UK GDP growth are available up to 24 days after the end of the quarter for which a nowcast is required. For the Euro Area preliminary estimates are published 45 days after the respective quarter.

${ }^{2}$ Apart from Aruoba et al. (2009) who also include series in weekly frequency, these studies focus on indicators in monthly and quarterly frequency.

${ }^{3}$ As an alternative track Castle and Hendry (2010) advanced an approach based on bridging equations (as interpolating devices). It addresses model uncertainty by automatic model selection including an impulse-indicator saturation (IIS) algorithm.
} 
variables, the idea is to enlarge this set. The decision of adding a new indicator is based on whether its inclusion increases the percentage of variance of GDP growth explained by the common factor or not. This method is intended to lead to a "screening out of those additional indicators that capture idiosyncratic dynamics and that do not lead to better fit for GDP growth through the common component." However, this method has a crucial drawback as the ordering with which new indicators are added and possibly screened out is arbitrary. It might be the case that one candidate indicator substantially increases the percentage of variance of GDP growth explained by the common factor when considered as ultimate or penultimate indicator (after several new candidate indicators having been included) but does not lead to an improvement of the fit of the common factor if considered as first candidate. Camacho and Perez-Quiros (2010, p. 674) are aware of this deficiency by noting that they are not treating newly added variables symmetrically and that further research regarding the implementation stage of using a DFM for nowcasting purposes is needed.

The present study contributes to the improvement of the implementation stage of approximate DFMs. In order to address model uncertainty in the context of selecting indicators as input for an approximate DFM to nowcast GDP, we propose to subject the final step of the selection of indicators to an extreme bounds analysis (EBA) ${ }^{4}$ In a simple and stylized exemplary application, we show that including a basic EBA as final step in the selection process improves the accuracy of the nowcast. Our sample application consists in nowcasting Spanish GDP growth at a monthly frequency, which through official channels is available only in quarterly frequency. Our selection of indicators from a "universe of potentially available time series" (Camacho and Doménech, 2012) proceeds in two steps. First, we reduce a set of 258 time series related to Spanish GDP and available at monthly frequency to 27 series by keeping only those series showing a reasonable correlation with the series to be nowcasted both contemporaneously and at its quarterly and yearly lags. This allows us in a second step to apply an EBA procedure to narrow this set further by dropping "non-robust" or "fragile" indicators. To identify robust indicators, the strategy is to consider all possible regressions with quarterly GDP as dependent that can be estimated by taking combinations of a parsimonious sub-set of the 27 pre-selected series. The sensitivity analysis then consists in checking for each series whether the resulting distribution of parameter estimates has only positive or negative support, that is, whether the respective coefficient estimate does not change its sign in all regression runs. The latter should, at least, hold in an interval of so-called "extreme bounds" (Levine and Renelt, 1992). Following this strategy, we find 14 indicators reasonably correlated with GDP that are robust in explaining GDP growth according to these standards: Besides industrial production and a series of survey-based

\footnotetext{
${ }^{4}$ EBA was originally suggested by Leamer $(1983,1985)$ and Levine and Renelt (1992) in the context of choosing exogenous variables in cross-country growth regressions.
} 
confidence and order-book indicators, a real estate stock exchange index and the number of workers subject to social security contributions qualify.

\section{Approximate Dynamic Factor Model}

The starting idea of DFMs is the assumption that each series of a set can be written as the sum of two stochastic components: a common factor $f_{t}$, which for instance reflects the overall business cycle component of an economy and an idiosyncratic component $u_{t}$ which captures the specific characteristics of each series (Lütkepohl, 2005). The sum of the two components can be written as:

$$
y_{t}=L f_{t}+u_{t},
$$

where $y_{t}$ is a $K \times 1$ vector of variables, $f_{t}$ is an $N \times 1$ vector of common factors with $N<K$, and $L$ is a $K \times N$ matrix of factor loadings. Factor analysis models assume that the idiosyncratic components $u_{t}, u_{t-s}$ are independent. The same applies to factors $f_{t}, f_{t-s}$. However, for estimating time series it is more realistic to allow for some serial correlation of factors. Thus, equations for the common factor $f_{t}$ and the idiosyncratic component $u_{t}$ are more reasonably specified as

$$
f_{t}=A_{1} f_{t-1}+\cdots A_{p} f_{t-p}+\eta_{t}
$$

and

$$
u_{t}=C_{1} u_{t-1}+\cdots C_{q} u_{t-q}+\varepsilon_{t},
$$

where $\eta_{t}$ and $\varepsilon_{t}$ represent white noise processes.

The coefficients to be estimated are the factor loadings coefficient matrix $L$ and matrices $A_{i}$ and $C_{i}$ for $i=1, \ldots, p$.

Finally, the equation used to forecast GDP growth rate $\gamma$ some $h$ periods ahead is written as

$$
\gamma_{t+h}=\mu+a(L) \gamma_{t}+b(L) \widehat{f}_{t}+e_{t+h},
$$

where $a(L)$ and $b(L)$ denote lag polynomials in $L$ of order $p_{a}$ and $p_{b}$, respectively, i.e. $a_{0}+a_{1} L+\ldots+a_{p_{a}} L^{p_{a}}$ and $b_{0}+b_{1} L+\ldots+a_{p_{b}} L^{p_{b}}$; see Breitung and Eickmeier (2006). Stock and Watson (2010) review three generations of different DFM estimation methods. The estimation procedure in this study follows closely the one used by Camacho and Perez-Quiros (2010), Camacho and Garcia-Serrador (2013) and Camacho and Doménech (2012) which might be classified to belong to the first generation (time-domain maximum likelihood via the Kalman filter) of approximate DFMs, which is particularly parsimonious as regards the small-scale property of models.

The actual estimation comprises three steps. The first one is the computation of the 
Gaussian Likelihood using the Kalman Filter. The second one consists of the estimation of the parameters of matrices $L, A_{i}$ and $C_{i}$. The last step is the utilization of the Kalman Filter smoother to obtain estimates of factor $f_{t}$.

The Kalman Filter recursively computes unobserved states given certain observations minimizing the mean of the squared error. As equation (1) contains the available information it represents the observation equation, while equations (2) and (3) model the unobserved state. Hence, they make our state equations in the state-space representation. The estimation proceeds in two stages: prediction and updating. Following Stock and Watson (1991), the first stage consists in the prediction equations which are given by

$$
\alpha_{t \mid t-1}=T_{t} \alpha_{t-1 \mid t-1}
$$

where $\alpha_{t \mid \tau}$ represents the estimate of the vector of states ( $f_{t}$ and $u_{t}$ in this case) according to the available information of the indicators of vector $y$ at all periods until $\tau$, and

$$
P_{t \mid t-1}=T_{t} P_{t-1 \mid t-1} T_{t}^{\prime}+Q_{t}
$$

where $P_{t \mid \tau}=E\left[\left(\alpha_{t \mid \tau}-\alpha_{t}\right)\left(\alpha_{t \mid \tau}-\alpha_{t}\right)^{\prime}\right]$, that is the estimation of the covariance matrix of the state vector with the available information until period $\tau$.

Given this information, the vector of indicators $y_{t}$ can be predicted at period $t-1$ by

$$
y_{t \mid t-1}=L \alpha_{t \mid t-1},
$$

and the implied forecast error is given by

$$
v_{t \mid t-1}=y_{t}-L \alpha_{t \mid t-1}
$$

The corresponding variance-covariance matrix is

$$
E\left[v_{t} v_{t}^{\prime}\right]=F_{t}=L P_{t \mid t-1} L^{\prime}
$$

Equations (5) to (9) are used to obtain a-priori estimates of values for state $\alpha_{t}$ given some arbitrary initial values $\alpha_{0 \mid 0}$ and $P_{0 \mid 0}$. Following the a-priori estimate, an a-posteriori update is calculated via

$$
\alpha_{t \mid t}=\alpha_{t \mid t-1}+P_{t \mid t-1} L^{\prime} F_{t}^{-1} v_{t}
$$

and

$$
P_{t \mid t}=P_{t \mid t-1}-P_{t \mid t-1} L^{\prime} F_{t}^{-1} L P_{t \mid t-1} .
$$

Proceedingly, the updated values for $\alpha_{t}$ and $P_{t}$ are used to re-do the same procedure recursively again. From there, the log likelihood is computed and maximized in order 
to estimate coefficients.

Obviously, the elementary and first challenge for the specification of the model is the selection of the indicators, i.e., of the variables contained in vector $y$.

\section{$2.1 \quad$ In-sample analysis}

The variable to be nowcasted for our sample case is quarterly GDP growth for the Spanish economy. The series is stationary and available in quarterly frequency between 1975 and 2011 (see solid line in Figure 1, depicted on left ordinate). To calculate the

factor $\hat{f}_{t}$ a group of indicators $\left(y_{t}\right)$ driven by the same common factor as the growth rates of GDP must be selected. Standard indicators capturing the economic expectations on production such as the business climate indicator for Germany provided by the ifo institute (IFO), the Economic Sentiment Indicator (ESI), and the Purchasing Managers confidence Indexes (PMI) for the Euro Zone have been used in recent works. Of course, there are myriads of indicators with potential explanatory power or nowcasting potential which one can think of besides confidence or climate indicators.

\subsubsection{Implementation}

The central contribution of this study is to mechanize the indicator selection process relying on correlation coefficient thresholds and the EBA approach. In our exemplary case, we initially consider 258 variables related to Spanish GDP. These variables contain expectation surveys on production data (national and regional), order book information, confidence indicators, electricity consumption, consumer surveys, social security and unemployment series, information on self-employment and state expenses, tax payments, bond yields, and other financial variables. All of these variables are available in a monthly frequency and cover, at least, 10 years during the period at stake. All non-deseasonalized series were seasonally adjusted using standard techniques. Time series which were not stationary in levels were transformed into monthly growth rates. All monthly growth rates were tested to be stationary.

Choice of core-set of indicators. The first criterion of selecting from variables intended to serve as indicators for the calculation of the common factor is the correlation coefficient with the GDP quarterly growth rate. We consider the respective coefficients for each series as well as its quarterly and yearly lags. To qualify for the core-set in contemporary or lagged expression it is required to top a threshold for the respective correlation coefficient amounting to $|.05|$, i.e. 5 percent in absolute terms. In our sample nowcast of monthly GDP growth rates for Spain, 27 series meet this requirement.

Extreme bounds analysis. The second part of our proposal for a selection procedure consists in applying a standard EBA (Levine and Renelt, 1992) in order to check if the selected variables are "robust" in explaining GDP growth. The central idea of this 
part of the procedure is to check whether the remaining $K$ candidate indicators (here, $K=27)$ for the common factor are truely stable in their ralationship with GDP growth. To this end, for each of the $K$ candidate variables $c$ a total number of $J$ of the following regressions are estimated

$$
\gamma_{t}=\delta_{j}+\beta_{c_{j}} c_{t}+\sum_{i=1}^{k} \beta_{x_{i, j}}(L) x_{t, i, j}+\varepsilon_{t, j}
$$

where $x_{i, j}$ denotes a vector of up to three $(k=3)$ additional indicator candidates, acting as randomly assigned conditioners in each of the $J$ regression models. The order $p_{x_{\beta}}$ of the polynomial $\beta_{x_{i, j}}(L)$ in $L$ is chosen on the basis of information criteria AIC and BIC for each $k$, respectively. Hence, the minimum number of models to be checked $J^{\text {min }}$ is

$$
J^{\min }=\left(\begin{array}{c}
K-1 \\
k
\end{array}\right)=\frac{(K-1) !}{k !(K-1-k) !}
$$

In our case $(K-1=26 ; k=3), J^{\text {min }}=2,600$ and, hence, a total number $K \times J^{\text {min }}=$ $27 \times 2,600=70,200$ would have to be estimated disregarding $L$. Cosindering $L$, we actually estimated a total of $K \times J=309,960$ models. For each model $j$, we obtain estimates $\widehat{\beta}_{c_{j}}$ and $\widehat{\sigma}_{\widehat{\beta}_{c_{j}}}$ distributed across regressions $j=1, \ldots, J$. Additionally, we define an upper and lower extreme bound $(E B)$ for each candidate indicator $c$ and for each model $j$, that is,

$$
\widehat{\beta}_{c_{j}} \pm 2 \widehat{\sigma}_{\widehat{\beta}_{c_{j}}} .
$$

A candidate indicator is said to be robust in the sense of Levine and Renelt (1992) if for all models $J$

$$
\operatorname{sign}\left(E B_{L}\right)=\operatorname{sign}\left(\widehat{\beta}_{c_{j}}-2 \widehat{\sigma}_{\widehat{\beta}_{c_{j}}}\right)=\operatorname{sign}\left(E B_{U}\right)=\operatorname{sign}\left(\widehat{\beta}_{c_{j}}+2 \widehat{\sigma}_{\widehat{\beta}_{c_{j}}}\right)
$$

in contrast, it is identified as fragile if

$$
\operatorname{sign}\left(E B_{L}\right)=\operatorname{sign}\left(\widehat{\beta}_{c_{j}}-2 \widehat{\sigma}_{\widehat{\beta}_{c_{j}}}\right)<0<\operatorname{sign}\left(E B_{U}\right)=\operatorname{sign}\left(\widehat{\beta}_{c_{j}}+2 \widehat{\sigma}_{\widehat{\beta}_{c_{j}}}\right) .
$$

The intuition behind applying EBA in the present context is straightforward: whether a candidate indicator $c_{t}$ behaves procyclically or countercyclically, i.e. is positively or negatively related with $\gamma_{t}$, is a defining property. This property should not change by including other indicator series as covariates, i.e. should not depend on whether $\gamma_{t}$ is subjected to a randomly drawn set of further indicators in contemporaneous or contemporaneous and lagged expressions. In our sample case, the above sketched EBA procedure identifies 14 variables as robust. They are listed in Table 1.

The selected variables contain mainly information about the industrial sector and expectations about the (near) future development of the economy. Industrial production of consumer goods, capital goods, and intermediate goods as well as order book 


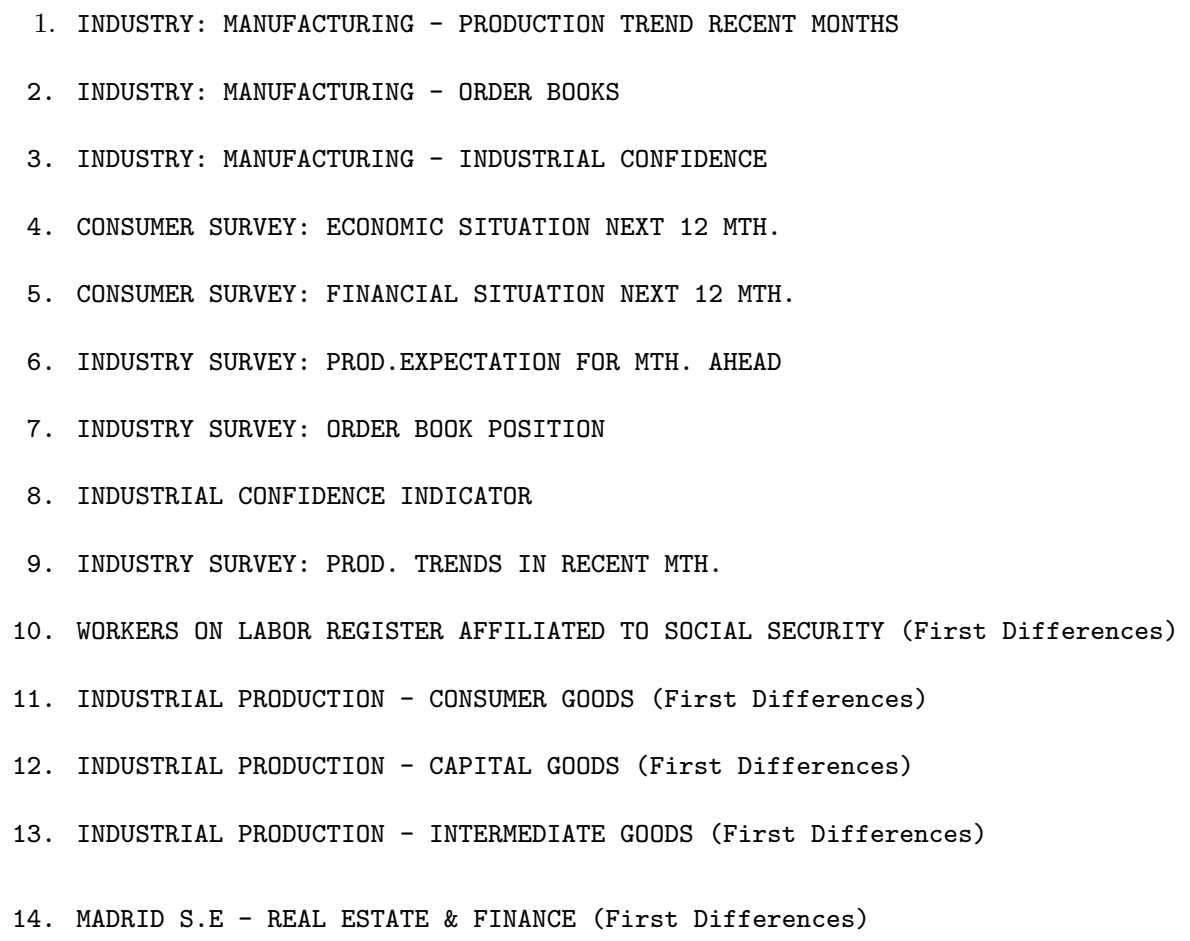

Table 1: Selected variables to calculate the common leading factor

assessments, industrial confidence and deviations of production in the manufacturing sector from its trend (production trend) in recent months turn out indicative -with a respective contemporaneous, leading, or lagging property that does not change with the inclusion of additional conditioners- in explaining Spanish GDP growth. Analogously, expectations about the economic and financial situation in the short-run (12 months) and in the very short-run (1 month) qualify as nowcasting indicators of GDP growth, respectively.

Two further selected variables are noteworthy. The first one is the number of "workers on labor register affiliated to social security" that indirectly also captures registered unemployment as a kind of "other side of the same coin" variable. The latter is typically identified as a central indicator in approximate DFM nowcasts of Spanish GDP 5 The second selected variable that stands out is the Madrid Stock Exchange for Real Estate and Finance Index. In the Spanish case, boom and bust dynamics on the real estate market, in particular, in the last two decades, are reasonable to be closely related to the dynamics of GDP growth. We find the most profound correlation of this index with GDP growth at a lag of eight months. Hence, we select the market value of real estate eight months ago as stable indicator for deducing Spanish GDP growth today.

\footnotetext{
${ }^{5}$ The authors owe this information to a remark by Domenico Giannone.
} 


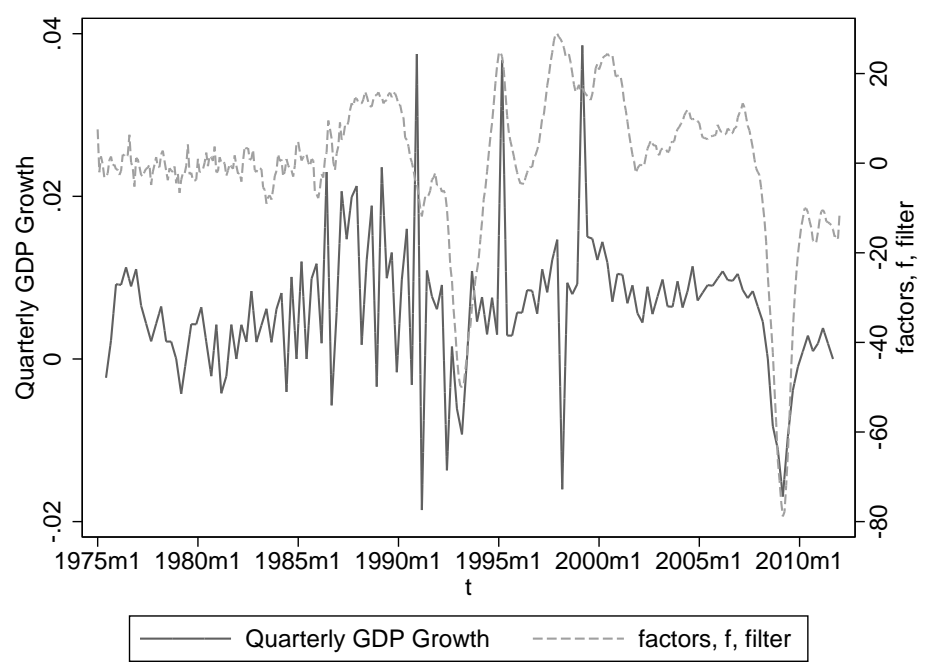

Figure 1: Estimated common factor $\hat{f}$ and quarterly GDP growth

Following this selection of indicators for the common factor, we proceed by estimating different DFMs in order to achieve a "best specification" of the model regarding the lag structure of state variables. To this end, we standardize all series to show zero mean and unity variance. This is due to handling shortfall in the series following Camacho and Perez-Quiros (2010), Camacho and Garcia-Serrador (2013) and Camacho and Doménech (2012) who refer to Mariano and Murasawa (2003) by replacing missing values due to mixed frequency and/or due to release delay of data (e.g. for industrial production) by random draws from a standard normal distribution. This strategy allows us to estimate DFMs considering different lag structures, while the estimation of the common factor by the Kalman Filter is not affected.

The final DFM specification, corresponding to lowest information criteria values, is obtained for lag orders $p=2$ and $q=1$ for $f_{t}$ and $u_{t}$, respectively ${ }_{6}^{6}$

\subsubsection{Absolute and relative performance: in-sample}

In Figure 1 the estimated common factor is plotted against the Spanish quarterly GDP growth series. The factor loadings behind are all estimated as significantly different from zero at standard levels. The graph shows that the common factor captures the cyclical movements of GDP as well as major expansions and contractions of quarterly growth rates fairly well. Extreme crises as the one in 1994 or the "great recession" starting at the end of 2008 are also accurately reflected by the indicators constituting the common factor. The crucial improvement is the monthly frequency of the derived factor. Most decisively, the common factor is calculated also for the last period of the

\footnotetext{
${ }^{6}$ See equations 22 and $(3)$; as maximum lag orders $p_{\max }=q_{\max }=2$ was chosen.
} 


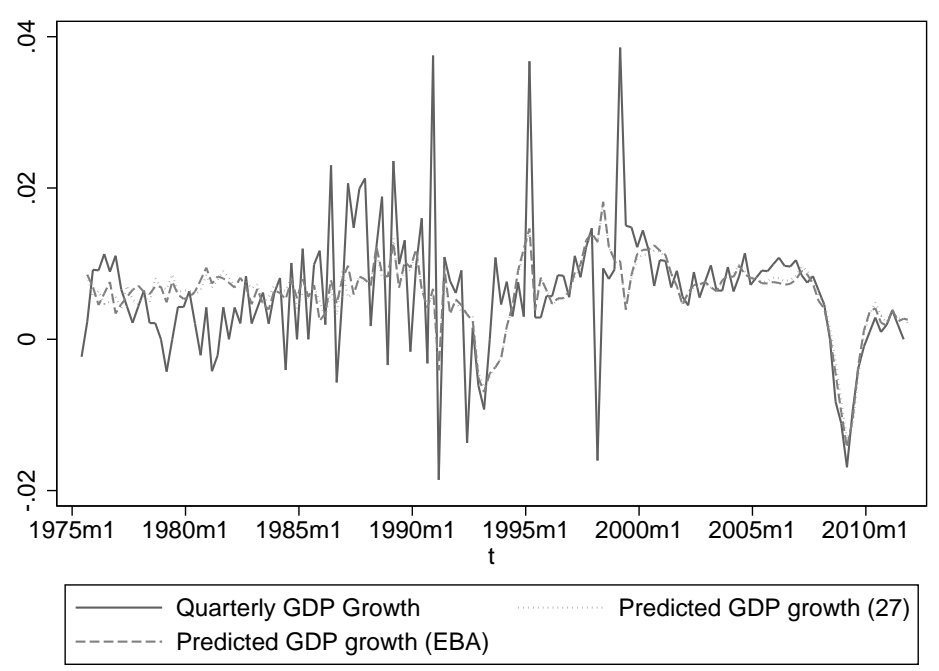

Figure 2: Predicted GDP growth $-\gamma_{t+h}=\mu+a(L) \gamma_{t}+b(L) \hat{f}_{t}+e_{t+h}$

sample, i.e. for the period in which GDP growth is unknown and of central interest for decision makers.

The estimated set of common factor $\hat{f}_{t}$ is the final ingredient needed to determine the adequate lag structure of the forecast equation $\gamma_{t+h}=\mu+a(L) \gamma_{t}+b(L) \hat{f}_{t}+e_{t+h}$. Trying all possible combinations from one to twelve lags (one year) for lag polynomials $a(L)$ and $b(L)$ for equation (4) and dropping insignificant lags, we find the one in accordance with lowest information criteria values to be

$$
\gamma_{t+h}=\mu+\alpha_{0} \gamma_{t}+\alpha_{3} \gamma_{t-3}+\beta_{0} \hat{f}_{t}+\beta_{1} \hat{f}_{t-1}+\beta_{2} \hat{f}_{t-2}+e_{t+h}
$$

Figure 2 shows observed and predicted values of Spanish GDP growth at quarterly frequency since 1975. Forecasted values represented by the dashed line were obtained following the above sketched EBA-robust selection of 14 out of 27 core indicators using the forecast equation given above. Particularly remarkable is the accuracy of the model in capturing not only the bust of the recent recession but also the turning point some months later. We conjecture the real estate price measure, that is, the central indicator from the sector from which the Spanish credit-boom cycle actually emanated, to be responsible for this exceptional performance. The lower schedule of Figure 2 also gives a first glimpse of the relative performance of the single-index derived on the basis of the full set of 27 core indicators (before applying the EBA selection step) against the one of the corresponding single-index derived on the basis of 14 variables from the core set after screening out fragile indicators relying on the above sketched EBA procedure. Obviously, the in-sample performance difference is of minor order, although a close 


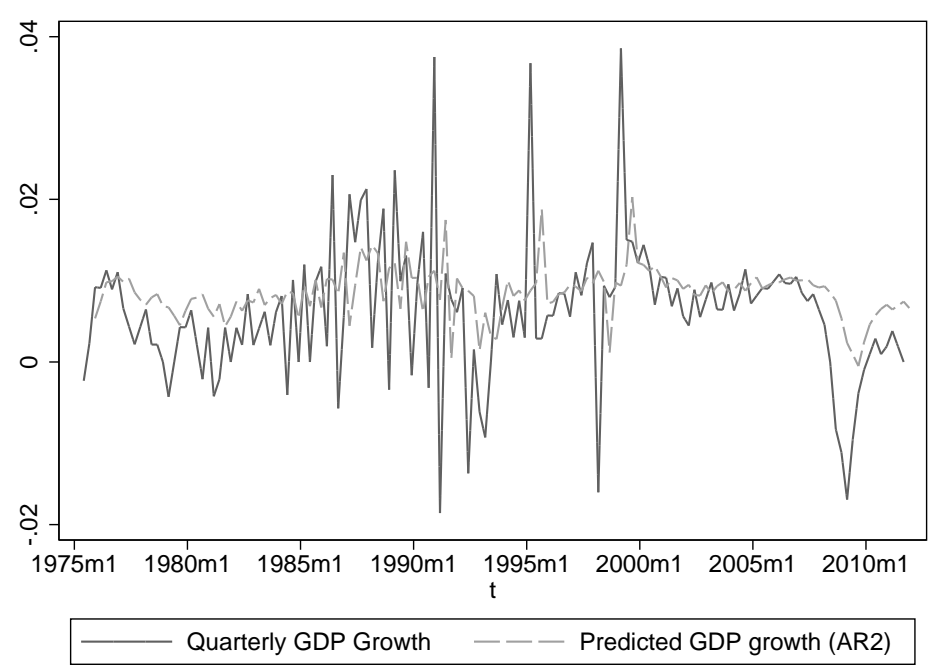

Figure 3: Predicted quarterly GDP growth - benchmark $A R(2)$ model

inspection reveals some differences, in particular, for calm phases of the cycle.

\section{Absolute and relative performance: out-of-sample}

To examine and compare the out-of-sample characteristics of the two indices based on the full set of 27 core indicators on the one hand and on 14 variables from the core set after screening out fragile indicators using EBA techniques on the other, we consider an $A R(2)$ model as benchmark predictor. The in-sample predicted GDP growth obtained from a benchmark $A R(2)$ process along with actual quarterly GDP growth figures is shown in Figure 3 above.

We proceed widely in line with the applied literature (see, e.g., Castle et al., 2009) in judging the out-of-sample forecast potential of the derived common factor. In order to assess the one-period ahead forecast properties, we treat the period starting the second quarter of 1975 to the first quarter of 2003 as given and successively extend this window by an additional quarterly observation to make one-quarter ahead projections for each successive step of prolonging the in-sample- "window" up to the penultimate quarter. Hence, our first projection uses equation (4) with $h=1, t=1$ (1975:Q2), .., $T_{1}$ (2003:Q1), and (justified by information criteria) $p_{a}=p_{b}=2$ to make a respective forecast of the GDP growth rate $\gamma_{T_{1}+1}^{F}$. We repeat this procedure for $T_{2}$ (2003:Q2) to $T_{34}$ (2011:Q2) in order to obtain a series of one-step ahead forecasts $\gamma_{T_{1}+1}^{F}, \ldots, \gamma_{T_{34}+1}^{F}$. For the univariate benchmark $A R(2)$ predictor $a(L)^{\prime}=\left(\begin{array}{lll}1 & 0 & 0\end{array}\right)$, while $b(L)^{\prime}=\left(\begin{array}{lll}0 & 0 & 0\end{array}\right)$.

From $\gamma_{T_{1}+1}^{F}, \ldots, \gamma_{T_{34}+1}^{F}$ we can calculate absolute forecast errors (AFE) for $\widehat{f}_{t}$ derived without EBA, i.e. AFE (27), and with EBA, i.e. AFE (EBA), respectively. Results 
along with the AFE of the benchmark $A R(2)$ are shown in the upper part of Figure 4. The dashed line refers to the EBA-robustly implemented DFM derived index based on 14 constituting indicators, the solid line to the one based on 27 constituting indicators, and the dotted one to the benchmark $A R(2)$ predictor, respectively. Obviously, both DFM-based models generate much lower forecast errors than the benchmark $A R(2)$. In particular, this holds for times of crises. The error measure spike at the beginning of the great recession and at its onset is the lowest for the EBA-robust DFM index. Even just by eyeballing, we get the impression that the more parsimonious EBA based model slightly outperforms the one based on nearly double as much, that is, 27 indicator series. Putting a stronger weight on larger deviations of forecasts from actual values, we obtain a similar picture for the squared forecast errors (SFE) as shown in the lower part of Figure 4.

Figures of these forecast errors averaged for different periods are summarized in Table 2, where "relative" errors refer to ratios of the respective errors to the corresponding errors of the $A R(2)$ benchmark model.

\begin{tabular}{lccc}
\hline & Without EBA & With EBA & AR(2) \\
Total period (averaged one-step forecast errors) & & & \\
\hline SFE & 0.0012 & 0.0008 & 0.0043 \\
Relative SFE & 0.2736 & 0.1966 & - \\
AFE & 0.0025 & 0.0021 & 0.0039 \\
Relative AFE & 0.6466 & 0.5484 & - \\
Excluding 2008:Q2 to 2011:Q4 & & & \\
\hline SFE & 0.0005 & 0.0003 & 0.0006 \\
Relative SFE & 0.8274 & 0.4234 & - \\
AFE & 0.0018 & 0.0013 & 0.0018 \\
Relative AFE & 1.0082 & 0.7146 & - \\
Excluding 2008:Q3 to 2009:Q2 & & & \\
\hline SFE & 0.0007 & 0.0006 & 0.0008 \\
Relative SFE & 0.9406 & 0.8007 & - \\
AFE & 0.0021 & 0.0013 & 0.0018 \\
Relative AFE & 0.9794 & 0.8576 & - \\
\hline \hline
\end{tabular}

Table 2: Forecast errors across models 

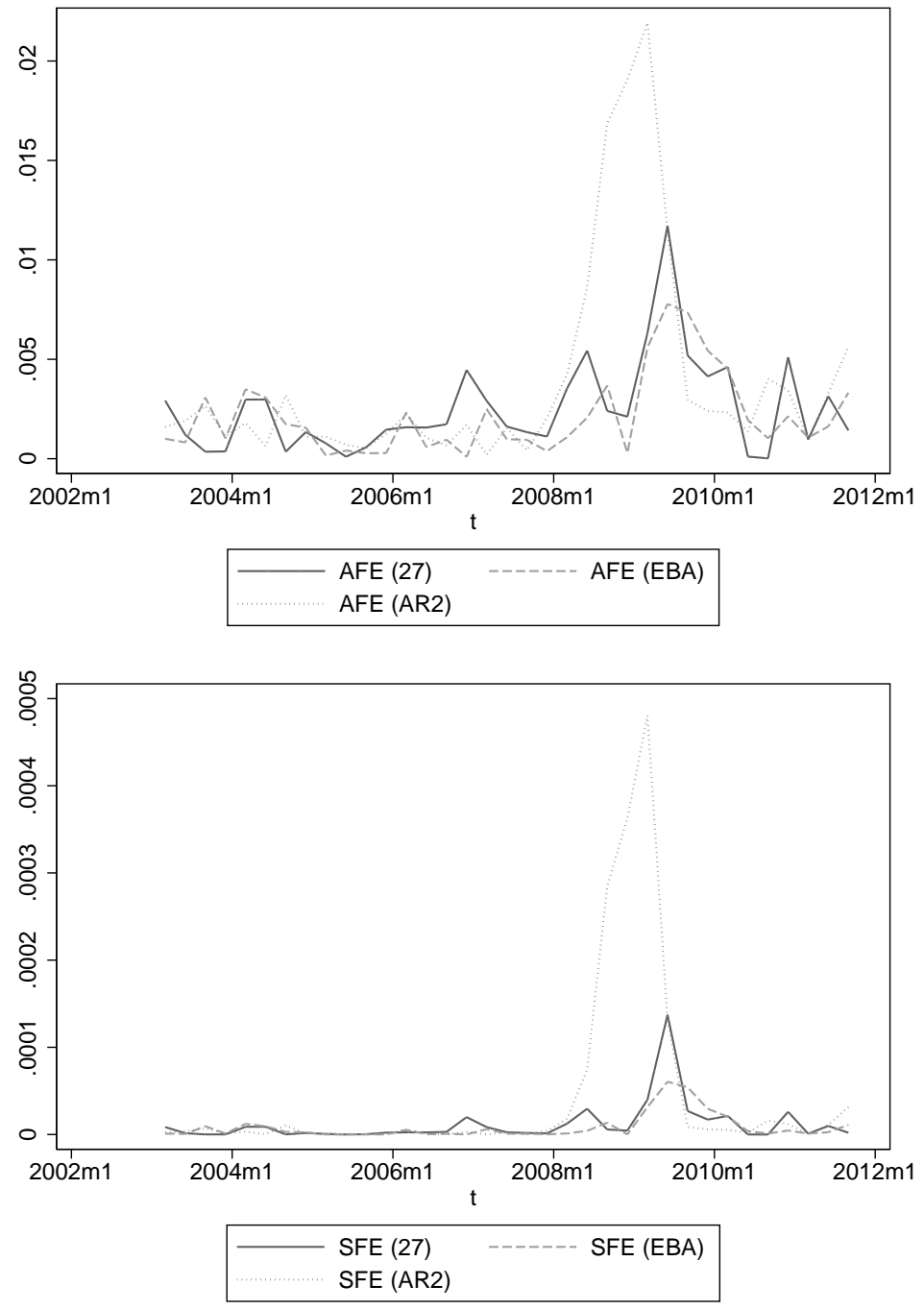

Figure 4: Absolute Forecast Error (AFE) and Squared Forecast Error (SFE) at each predicted period, based on rolling window starting first quarter 2003 

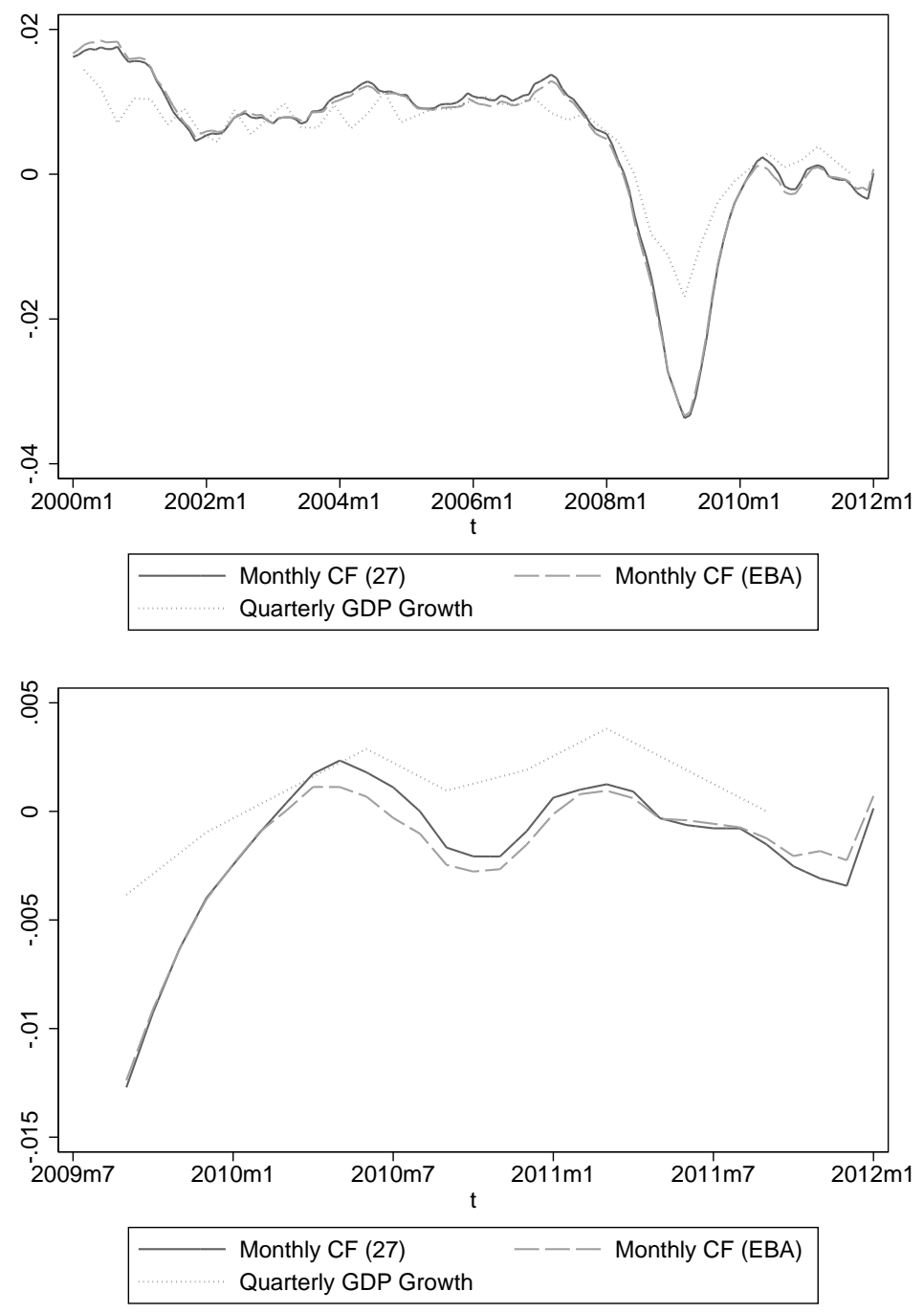

Figure 5: Monthly common factors $(\mathrm{CF})$ and quarterly GDP growth rates; at end of observation period: 2000s and mid-2009 to 2012 


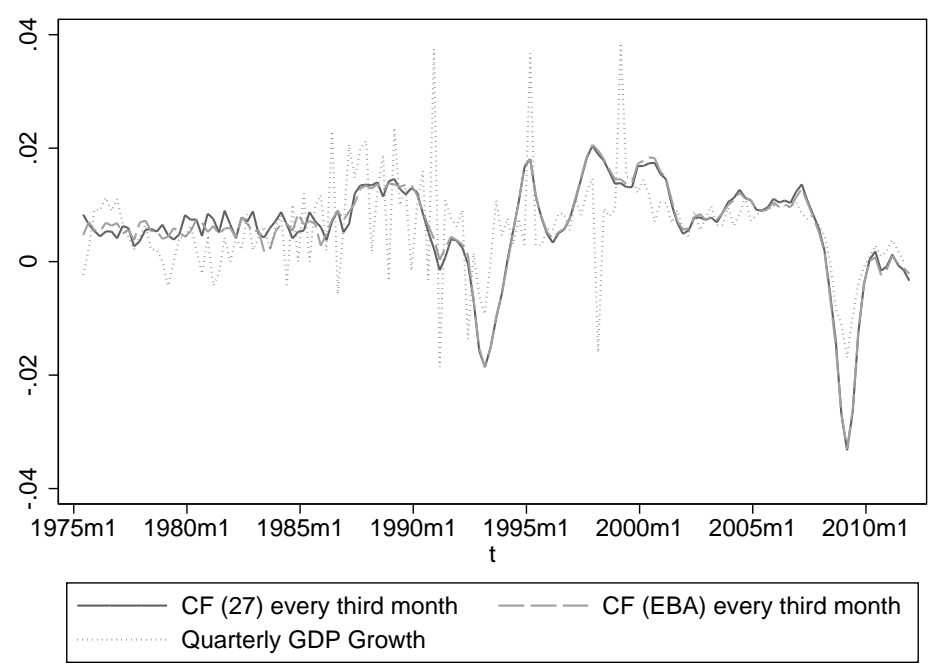

Figure 6: Common factors $(\mathrm{CF})$ and quarterly GDP growth

Without EBA With EBA Difference

Total period

\begin{tabular}{lccc}
\hline MANE & 0.6492 & 0.6396 & -.0096 \\
MSNE & 0.9827 & 0.9846 & 0.0019 \\
Excluding 2008:Q2 to 2011:Q4 & & & \\
\hline MANE & 0.5384 & 0.5261 & -.0123 \\
MSNE & 0.5785 & 0.5808 & 0.0023 \\
Excluding 2008:Q3 to 2009:Q2 & & & \\
\hline MANE & 0.5870 & 0.5768 & -.0102 \\
MSNE & 0.7109 & 0.7120 & 0.0011 \\
\hline \hline
\end{tabular}

Table 3: Nowcast errors across models

Obviously, the common factor extraction including an EBA in the final pre-selection stage outperforms the one excluding it across all considered measures and periods shown in Table 2. We consider for the averaging of forecast errors the total forecast period from $T_{1}$ (2003:Q1) to $T_{34}$ (2011:Q2) as well as both a period excluding the complete period following the great recession and a period that leaves out the quarters from the third quarter of 2008 to the second quarter of 2009, that is, the period when the shock corresponding to the crisis hit the economy 7 Our results remain mainly untouched.

\footnotetext{
${ }^{7}$ One reason for doing so is that one might be bothered that by merely dropping some indicators in the derivation of the common factor raises the weight of the stock exchange for real estate and finance index in the constitution of the factor and through this channel alone
} 
Throughout forecast erros are the lowest for the common factor including the EBA exercise in the final step of its implementation. It is also this factor that throughout ourperforms the univariate benchmark model by implying relative forecast errors $<1$.

Assessing only the one-period ahead forecasting potential using rolling window techniques does, however, not fully measure up to the potential of approximate DFM derived factors to nowcast politically relevant economic series as outlined in the introductory paragraphs of the present study. We, therefore, also suggest to examine the nowcasting properties of the derived monthly indices over the entire period of investigation. A visual impression is given in Figure 6, while Figure 5 highlights the dynamics of the common factors in monthly frequency vis-à-vis the quarterly GDP growth rates in the particularly critical period of the 2000s as well as for the mid-2009 to 2012 period. An inspection of the right-end of series in the lower part of Figure 5 highlights the actual nowcasts of the analyzed indicators at monthly frequency. For these type of comparisons, the monthly common factor series were transformed to imply the same first and second moments as the actual GDP growth series. A summary of mean absolute nowcast errors (MANE) and mean squared nowcast errors (MSNE) of common factors transformed along these lines and evaluated at every third observation (month) is given in Table 3. The EBA including common factor outperforms only as regards the absolute nowcast errors. However, with regard to MSNE values the differences to the ones of the 27 indicators based factor are much smaller and actually close to zero.

\section{Conclusion}

In this study, we analyzed the implementation stage of approximate dynamic factor models (DFMs) to nowcast GDP growth in a sample exercise using Spanish time series. We propose a two-tiered procedure to meachnize the implementation of parsimonious nowcasting models. In a first step, variables highly correlated with GDP are identified as "core" indicators. In a second step, they are checked for robustness in the sense of extreme bounds analysis before we use them in a DFM-framework to exemplarily nowcast Spanish GDP growth. We find that our implementation produces more accurate nowcasts than both a benchmark stochastic process and the implementation based on the total set of core indicators. It remains for future work to refine the suggested meachnization which, as we are aware, still has a somewhat "brute force" character. Additionally, we leave it to follow-up work to scrutinize both stages of the proposed procedure with regard to their stability in screening out indicators over different recursively enlarged sub-period samples. Nevertheless, the analyzed back-of-the-envelope strategy already outperformed both a basic autoregressive process

gives the EBA based factor a comparative advantage. However, as can be seen from Table 2, the AFE difference between the two factors is the largest if we leave out the recent crisis which is generally seen to be rooted in Spanish housing price dynamics. 
and a less parsimonious model as regards predictive capability. Finally and most importantly, the proposed procedure overcomes a common deficiency of the existing literature by treating candidate indicators for the derivation of the common factor symmetrically against the backdrop of model uncertainty.

\section{References}

Angelini, E., Camba-Mendez, G., Giannone, D., Reichlin, L., and G. Rünstler, 2008a. Short-term Forecasts of Euro GDP Growth, CEPR Discussion Paper, No. 6746 .

Angelini, E., Banbura, M., and G. Rünstler, 2008b. Estimating and Forecasting the Euro Area Monthly National Accounts from a Dynamic Factor Model, ECB Working Paper, No. 953.

Aruoba, S., Diebold, F.X., and C. Scotti, 2009. Real-time Measurement of Business Conditions, Journal of Business and Economic Statistics 27, 417-427.

Breitung, J. and S. Eickmeier, 2006. Dynamic Factor Models, Advances in Statistical Analysis 90, 27-42.

Camacho, M. and R. Doménech, 2012. MICA-BBVA: A Factor Model of Economic and Financial Indicators for Short-term GDP Forecasting, Journal of the Spanish Economic Association 3, 475-497.

Camacho, M. and A. Garcia-Serrador, 2013. The Euro-STING Revisited: The Usefulness of Financial Indicators to Obtain Euro Area GDP, forthcoming in Journal of Forecasting.

Camacho, M. and G. Perez-Quiros, 2010. Introducing the Euro-STING: Short-term Indicator of Euro Area Growth, Journal of Applied Econometrics 25, 663-694.

Castle, J.L., Fawcett, N.W.P., and D.F. Hendry, 2009. Nowcasting is not just Contemporaneous Forecasting, National Institute Economic Review 210, 71-89.

Castle, J.L. and D.F. Hendry, 2010. Nowcasting from Disaggregates in the Face of Location Shifts, Journal of Forecasting 29, 200-214.

Leamer, E.E., 1983. Let's Take the Con out of Econometrics, American Economic Review 73, 31-43.

Leamer, E.E., 1985. Sensitivity Analysis Would Help, American Economic Review 75, 308-313. 
Levine, R. and D. Renelt, 1992. A Sensitivity Analysis of Cross-country Growth Regressions, American Economic Review 82, 942-963.

Lütkepohl, H., 2005. New Introduction to Multiple Time Series Analysis, Springer: Heidelberg, New York

Mariano, R.S. and Y. Murasawa, 2003. A New Coincident Index of Business Cycles Based on Monthly and Quarterly Series, Journal of Applied Econometrics 18, 427-443.

Stock, J.H. and M.W. Watson, 1991. A Probability Model of the Coincident Economic Indicators, in Lahiri, K. and G. Moore (eds.), Leading Economic Indicators: New Approaches and Forecasting Records, Cambridge University Press: Cambridge, 63-89.

Stock, J.H. and M.W. Watson, 2010. Dynamic Factor Models, in Clements, M.P. and D.F. Hendry (eds.), Oxford Handbook of Economic Forecasting, Oxford University Press: Oxford, 35-60. 